Recepción: 20 / 04 / 2017

Aceptación: 20 / 05 / 2017

Publicación: 15 / 07 / 2017

Ciencias Médicas

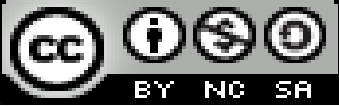

Artículo Científico

\title{
Incidencia de la hernia inguinal y la evolución en pacientes
}

\author{
Incidence of inguinal hernia and evolution in patients
}

\section{Incidência de hérnia inguinal e evolução em pacientes}

Rosa A. Rivas-Vásquez ${ }^{\text {I }}$ rosa.rivasv@ug.edu.ec

Ana M. Izurieta Enríquez II ana.izurietae@ug.edu.ec

Janet P. Emén-Sánchez III janet.emens@ug.edu.ec Gisella Ruiz-Ortega IV gisella.ruizo@ug.edu.ec

Correspondencia: rosa.rivasv@ug.edu.ec

Especialista en Cirugía General; Magister en Emergencias Médicas; Doctor en Medicina y Cirugía; Universidad de Guayaquil, Guayaquil, Ecuador.

II. Especialista en Cirugía General; Medico; Universidad de Guayaquil, Guayaquil, Ecuador.

III. Master Universitario en Nutrición Humana y Dietética Aplicada; Especialista en Medicina del Deporte; Médica; Universidad de Guayaquil, Guayaquil, Ecuador.

Iv. Magister en Atención Primaria y Clínica Infantil; Especialista en Cirugía Plástica Reconstructiva y Estética; Diploma Superior de Cuarto Nivel en Desarrollo Local y Salud; Magister en Gerencia en Salud para el Desarrollo Local; Doctor en Medicina y Cirugía; Universidad de Guayaquil, Guayaquil, Ecuador. 


\section{Resumen}

El objetivo de la presente investigación fue determinar por medio de revisión de historias clínicas la incidencia de la Hernia Inguinal y la evolución de los pacientes con reparación por medio de la Técnica TAPP en el Servicio de Cirugía General del Hospital Dr. Federico Bolaños Moreira en el periodo 2013- 2015. La metodología de investigación empleada fue un estudio de tipo longitudinal, retrospectivo no experimental para determinar la incidencia, factores de riesgo y los beneficios en cuanto a complicaciones tempranas y tiempo de hospitalización entre los pacientes. El universo utilizado para la presente investigación correspondió a un total 105 casos atendidos. Entre algunas de las conclusiones que se pudieron observar fue una distribución similar del defecto herniario en cuanto a lateralidad y distribución de género, siendo la región inguinal derecha y el sexo masculino las características de distribución más prevalentes, Los factores de riesgo que se identificaron con mayor frecuencia fueron el debilitamiento de tejidos de la pared relacionado a mayor edad y obesidad, Las ventajas de menor dolor y rápida recuperación convierten a la TAPP en una excelente opción siempre y cuando el cirujano cuente con suficiente experiencia con la técnica.

Palabras Claves: Hernia inguinal; TAPP; cirugía; hospitalización. 


\begin{abstract}
The aim of the present investigation was to determine, through a review of medical records, the incidence of Inguinal Hernia and the evolution of patients with TAPP repair in the General Surgery Service of the Dr. Federico Bolaños Moreira Hospital in the Period 2013-2015. The research methodology used was a retrospective longitudinal, non-experimental study to determine the incidence, risk factors and benefits in terms of early complications and time of hospitalization among patients. The universe used for the present investigation corresponded to a total of 105 cases attended. Among some of the conclusions that could be observed was a similar distribution of the hernia defect in terms of laterality and gender distribution, with the right inguinal region and the male sex being the most prevalent distribution characteristics. Risk factors that were identified with greater Frequencies were the weakening of wall tissues related to older age and obesity. The advantages of lower pain and rapid recovery make the TAPP an excellent option as long as the surgeon has sufficient experience with the technique.
\end{abstract}

Key Words: Hernia inguinal; TAPP; surgery; hospitalization. 


\section{Resumo}

O objetivo da presente pesquisa foi estabelecido por meio de revisão de historias clínicas a incidência da Hernia Inguinal e a evolução dos pacientes com reparo por meio da Técnica TAPP no Serviço de Cirurgia Geral do Hospital Dr. Federico Bolaños Moreira en el Período 2013-2015. A metodologia de pesquisa empregada foi um estudo de tipo longitudinal, retrospectivo não experimental para determinar a incidência, fatores de risco e os benefícios em quanto a complicações tempranas e tempo de hospitalização entre os pacientes. $\mathrm{O}$ universo utilizado para a presente investigação corresponde a um total de 105 casos atendidos. Entre as opções de divulgação e a divulgação de informações relacionadas à distribuição de gênero, bem como a lateralidade e distribuição de gênero, sendo a região inguinal direita e o sexo masculino, as características de distribuição mais prevalentes, Os fatores de risco que se identificaram com prefeito A frequência e o debilitamento de tecidos relacionados com a antecipação de idade e obesidade, as vantagens de menor dor e rápido, a fim de recuperar a capacidade da TAPP em uma excelente opção, sempre que o cirujano cuente com a experiência técnica.

Palavras-chave: Hernia inguinal; TAPP; cirugía; hospitalização. 


\section{Introducción}

Las hernias inguinales son frecuentes en la práctica clínica, afectando entre un 3 a $5 \%$ de la población general. En los últimos años ha pasado de ser considerado un tema poco importante a ser uno de los procesos mejor estudiados y donde la excelencia del tratamiento es el objetivo primario. (Pinzón, Rincón, \& Espitita, 2011) (Gao, Han, Tian, \& Yang, 2010) (Campanelli, Canziani, Frattini, Cavalli , \& Agrusti, 2008)

La hernia inguinal es la protrusión de un saco revestido de peritoneo a través de la capa musculo aponeurótica de la pared abdominal por un punto débil del conducto inguinal, su génesis está asociado a muchos factores predisponentes entre los que se pueden citar: aumento crónico de la presión intraabdominal y la debilidad muscular. La obesidad y ascitis son factores que favorecen las hernias. Otras causas que debilitan el tejido conjuntivo son el tabaquismo, el envejecimiento y algunas enfermedades sistémicas. (Voyles, 2003)

En el mundo se operan cada año alrededor de 2.000 .000 de hernias inguinales, representando gran parte de las intervenciones de la cirugía general. Se presenta con mayor frecuencia en hombres, 9:1 antes del año de edad y después de los 55 años. La incidencia de recidiva es variable dependiendo del centro asistencial, la técnica quirúrgica y la condición de los tejidos. (Mangram , Horan, Pearson, \& Silver, 1999)

La investigación actual pretende determinar la incidencia y factores predisponentes al desarrollo de hernia inguinal en los pacientes atendidos en el área de cirugía del hospital "Dr. Federico Bolaños Moreira" en el periodo 2013- 2015; además del beneficio de la técnica preperitoneal transabdominal laparoscópica (TAPP) en el manejo de pacientes con patología de hernia inguinal. Los resultados servirán para que el personal médico, las autoridades de la institución y de gobierno desarrollen 
programas y actividades que permitan emprender políticas de salud encaminadas a disminuir su incidencia y brindar el tratamiento quirúrgico oportuno en pacientes con hernia inguinal. (Patiño, García-Herreros, \& Zundel, 1998)

\section{Materiales y métodos}

No se dispone con facilidad de cifras seguras sobre la incidencia de hernias inguinales en el Ecuador, sin embargo el Ministerio de la Salud Publica en el 2012 registra 14.832 casos, El 25.55\% se registraron en la provincia del Guayas y la relación Hombre vs. Mujer fue del 2.67:1. Se estima que la incidencia de hernia inguinal en Ecuador como el resto del mundo oscila entre el 3 al $5 \%$ de la población general. Constituyéndose probablemente en un problema de salud pública.

En el hospital Dr. Federico Bolaños Moreira se observa un aumento significativo del número de pacientes con evidencia de hernia inguinal, sin que exista información estadística precisa y sean además identificadas factores que motiven este incremento. Por esta razón, es necesario plantear una investigación que nos permita en forma documentada establecer qué población se encuentra más vulnerable e identificar los factores predisponentes relacionados con la misma. Además siendo la técnica TAPP una de las opciones quirúrgicas viables y con excelentes resultados demostrados en diversos estudios, sería conveniente evaluar el beneficio en cuanto a complicaciones postoperatorias y la recuperación post quirúrgica del paciente. En vista de todo lo antes planteado se generan las siguientes interrogantes.

1) ¿Cuál es la distribución de la patología herniaria según edad y género?

2) ¿Cuáles son los tipos de hernias más frecuentes según su localización?

3) ¿Cuáles son los factores de riesgos para el desarrollo de la hernia inguinal? 
4) ¿Cuáles fueron las complicaciones post quirúrgicas tempranas más frecuentes?

5) ¿Cuál fue el tiempo de recuperación en los pacientes con diagnóstico de hernia inguinal que se sometieron a reparación por técnica TAPP?

En este contexto, la presente investigación fue un estudio de tipo longitudinal, retrospectivo no experimental para determinar la incidencia, factores de riesgo y los beneficios en cuanto a complicaciones tempranas y tiempo de hospitalización entre los pacientes atendidos en el área de cirugía general del Hospital Dr. Federico Bolaños Moreira IESS de Milagro.

El universo utilizado para la presente investigación correspondió a un total 105 casos atendidos, los cuales fueron revisados a los efectos de llegar a conclusiones ilustrativas en el marco de la presente investigación.

\section{Resultados}

Cuando se evalúa la distribución de las hernias inguinales según sexo y edad del paciente, en los resultados obtenidos, se puede observar que el promedio de edad de la muestra de pacientes que fueron sometidos a reparación a través de la TAPP en el área de Cirugía General del Hospital Dr. Federico Bolaños Moreira de Milagro fue de aproximadamente 50 años, lo cual está entre rangos similares a la casuística mundial; sin embargo, presenta una diferencia de menos 3 años, en comparación con otros estudios que reportan un promedio de edad de 53 años en países similares al nuestro. La mayor frecuencia hacia el sexo masculino en la presentación de casos también está en correlación con la casuística mundial, al igual que la relación hombre y mujer de 4:1.

Con respecto a los tipos de hernias inguinales más frecuentes por su localización los diversos tipos de hernias presentaron diferencias relacionadas al sexo y a la ubicación. Las hernias del lado 
derecho se presentaron en mayor cantidad $51.43 \%(n=54)$, con una preferencia para hombres $53.57 \%(n=45)$ vs. Mujeres 42.86\% (n=9). El grupo que le sigue es el de ubicación izquierda con $28.57 \%(\mathrm{n}=30)$, hombres $26.57 \%(\mathrm{n}=22)$ vs. Mujeres $38.10 \%(\mathrm{n}=8)$. Las hernias bilaterales se registraron con menos frecuencia, con un total de $20 \%(n=21)$, hombres $20.24 \%(n=17)$ y mujeres $19.05 \%(\mathrm{n}=4)$.

Referente a los factores de riesgo, se consiguió identificar en los pacientes con diagnóstico de Hernia inguinal que el mayor número se encontró a pacientes con debilitamiento de sus tejidos por la edad 36.1\% $(n=38)$, el segundo en importancia fue por obesidad $23.8 \%(n=25)$ de los cuales 21 tenían diagnóstico de sobrepeso (IMC: 25-29.9) y los 4 restantes sobrepeso grado I (IMC 30 a 34.9); el tercer grupo en importancia fue por antecedentes de cirugía previa $16.1 \%(\mathrm{n}=17)$, el cuarto grupo en importancia fue el factor herniogénicorelacionado a algún tipo de esfuerzo intenso de los músculos abdominales, ya sea en su actividad laboral o por algún deporte $12.3 \%$ (n=13), seguido de estreñimiento crónico $7.6 \%(n=8)$ y finalmente el menor grupo fue por Tabaquismo 3.8\% $(n=4)$.

Con respecto a las complicaciones postoperatorias tempranas que se presentaron en los pacientes con diagnóstico de hernia inguinal sometidos a reparación mediante la técnica TAPP, se encontraron 11 complicaciones $(10,47 \%)$ de un total de 105 pacientes, divididas de acuerdo al número de complicaciones en 4 (37\%) con dolor a nivel de puertos y en área inguinal (uno requirió re intervención quirúrgica para la extracción de clic), 2 (18\%) con hematoma (uno intraoperatorio por lesión de vasos epigástricos), 2 (18\%) pacientes con seroma (que no requirió ningún manejo solo observación), 2 (18\%) pacientes con neuralgia que fue de tipo transitoria (dentro de los primeros 15 días post cirugía desapareció, se manejó con hielo local y analgésicos)y 1 (9\%) con perforación intestinal. 
Para concluir el tiempo de estancia hospitalaria desde el ingreso hasta el día de alta del hospital fue de 48,3 horas, tomando en cuenta que ingreso fue 24 horas previo al procedimiento quirúrgico. El promedio general de tiempo quirúrgico fue de 110,3 minutos, tomando en cuenta que 20 minutos corresponden para la aplicación de la anestesia general y 15 minutos posterior a la cirugía hasta que se despierte el paciente. Es conocido que la duración del proceso quirúrgico tiende a disminuir cuando el equipo gana en experiencia El promedio de tiempo de incapacidad fue 8,3 días

\section{Recomendaciones}

Se recomienda continuar con nuevas investigaciones referente a este tema ampliándola a otros grupos etarios de nuestro país y en nuevas condiciones versus otras técnicas quirúrgicas para corroborar los resultados obtenidos en este trabajo de investigación lo que permitirá tener una perspectiva más adecuada de la problemática y encaminar acciones que disminuyan el impacto de esta patología sobre la población del Guayas y del País.

Fomentar el control periódico de salud y de vida activa a través de los centros primarios de atención.

Crear centros o multiplicar centros de actividad física con el objetivo de disminuir el sedentarismo, lo que elimina varios factores de riesgo que contribuyen a la aparición de nuevas patologías.

Incentivar en el personal médico el espíritu de actualización científica continua con el afán de mantener un alto índice de destreza en las técnicas quirúrgicas actuales para aplicarlos en pacientes con estas patologías herniarias; tomando en cuenta una curva de aprendizaje para estos procedimientos quirúrgicos. Cada institución debe mantener la infraestructura necesaria al día en los 
avances de las técnicas quirúrgicas, para poder brindar a nuestros pacientes una mejor alternativa de manejo de su patología.

\section{Conclusiones.}

Se pudo observar una distribución similar del defecto herniario en cuanto a lateralidad y distribución de género que en otras poblaciones a nivel mundial, siendo la región inguinal derecha y el sexo masculino las características de distribución más prevalentes.

En el promedio de edad de nuestra población (50 años) se observó una diferencia de menos 3 años, en comparación con otros estudios que reportan un promedio de edad de 53 años.

Los factores de riesgo que se identificaron con mayor frecuencia fueron el debilitamiento de tejidos de la pared relacionado a mayor edad y obesidad. Factores descritos en la literatura relacionados con la génesis de patología herniaria.

Frente a las complicaciones encontradas en el posoperatorio, el dolor a nivel de puertos y área inguinal corresponde a la más frecuente en nuestro estudio con el $37 \%$ referente al total de complicaciones y a un $4 \%$ con respecto al total de los pacientes operados.

Las ventajas de menor dolor y rápida recuperación convierten a la TAPP en una excelente opción siempre y cuando el cirujano cuente con suficiente experiencia con la técnica.

Se encontró una respuesta positiva por parte de los pacientes, con resultados favorables en diferentes aspectos, tanto en el procedimiento, estancia hospitalaria y la evolución postoperatoria.

En el análisis de datos no se incluye la valoración de la recurrencia dado que los registros de historias clínicas corresponden a los pacientes se han intervenido durante estos dos últimos años con 
esta técnica. Se requieren como un mínimo de 5 años para comenzar a evaluar recurrencias de la técnica.

No existen trabajos serios con un número suficiente de casos, tiempo mayores 5 años de evolución, y con parámetros suficientes para evaluar los costos de esta intervención y compararlos con los beneficios como son: el reintegro más pronto a las actividades laborales privadas, la esfera estética, menores complicaciones y menor número de recidivas.

\section{Bibliografía}

Campanelli, G., Canziani, M., Frattini, F., Cavalli , M., \& Agrusti, S. (2008). Inguinal hernia: State of the art. Int J Surg, 6(1), S26-8.

Gao, M., Han, J., Tian, J., \& Yang, K. (2010). Vypro II mesh for inguinal hernia repair: A meta analysis of randomized controlled trials. Ann Surg, 251(2), 838-842.

Mangram , A., Horan, T., Pearson, M., \& Silver, L. (1999). Guideline for Prevention of Surgical Site Infection. Am J Infect Control, 27(1), 97-132.

Patiño, F., García-Herreros, L., \& Zundel, N. (1998). Inguinal hernia repair. The Nyhus posterior preperitoneal operation. Surg Clin North Am, 78(1), 1063-1074.

Pinzón, F., Rincón, F., \& Espitita, E. (2011). Herniorrafia inguinal abierta versus laparoscópica y con nuevos materiales protésicos: análisis de su efectividad clínica y económica para el paciente, el hospital y el sistema sanitario. Rev Colomb Cir, 26(1), 242-259.

Voyles, C. (2003). Outcomes analysis for groin hernia repairs. Surg Clin North Am, 83(1), 1279-1287. 